\title{
Search of genome-wide associations for breeding of spring wheat varieties with high zinc content
}

\author{
Shepelev S.S. ${ }^{1 *}$, Shamanin V.P. ${ }^{1}$, Pototskaya I.V. ${ }^{1}$, Chursin A.S. ${ }^{1}$, Kuzmin O.G. ${ }^{1}$, \\ Morgounov A.I. ${ }^{2}$ \\ ${ }^{1}$ Omsk State Agrarian University, Omsk, Russia \\ ${ }^{2}$ Food and Agriculture Organization of the United Nations, Riyadh, Saudi Arabia \\ *email: ss.shepelev@omgau.org
}

Zinc is a biologically active essential trace element for human life and health, one of the sources of which is cereal grain. The search of loci that control the zinc accumulation in wheat grain with usage of GWAS serves as the basis for development of wheat breeding strategy for functional nutrition. A collection of 150 varieties and lines of spring bread wheat of various origins (varieties from Russia, Kazakhstan, USA, synthetic hexaploids with Aegilops tauschii genome) was used for GWAS. Phenotyping of this collection in 2017-2018 was carried out. Entries were distributed in plot that was area $1.4 \mathrm{~m}^{2}$. Field trials utilized a randomized complete block design with four replicates. The zinc content in grain was determined at the University of Nottingham (UK). Genotyping of entries at the University of Nebraska-Lincoln (United States) was performed using the iSelect90K array. The method FarmCPU was used in MVP R software package for association test of markers. GWAS revealed SNPs associated with high zinc content (MTAs) in A genome - 49 SNPs, B genome - 36 SNPs, D genome - 38 SNPs. In 2017 SNP markers identified: S1D_22099159, S7A_287882406, S5A_478857359, S2B_213037042, S2B_213037036. The most significant association with studied trait was revealed on chromosome 1D with effect $11.2 \mathrm{mg} / \mathrm{kg}$ (marker S1D_22099159). According to vegetation season results in 2018 nine MTAs were detected on chromosomes 4A, 5A, 6B，7B，2D $\left(\mathrm{p}<1 \times 10^{-6}\right):$ S7B_374441352, S2D_35548157, S2D_35548171, S6B_584715980, S6B_584715992, S5A_554563813, S2D_30935042, S2D_646433883, S4A_686579282. Marker S5A_554563813 was identified on chromosome $5 \mathrm{~A}$, which associated with zinc accumulation in grain $(41.0 \mathrm{mg} / \mathrm{kg})$. SNPs associated with high zinc content identified across two years $\left(\mathrm{p}<1 \times 10^{-4}\right)$ : S1D_481791231, S6A_597841993, S6D_451861634, S6D_451861645, S6D_451861651, S6D_451861655,S6A_597692159. Practical interest for breeding are synthetic wheat lines as sources of high zinc content in grain, and genes of D genome from the IWGSC RefSeq v1.0 were annotated as TraesCS1D02G059300, TraesCS1D02G187500, TraesCS2D02G018300, TraesCS3D02G098600, TraesCS5D02G437100, TraesCS6D02G359700. 